\title{
A MODIFIED GARCH MODEL WITH SPELLS OF SHOCKS
}

\author{
QINGFENG LIU ${ }^{1 \star}$ and KIMIO MORIMUNE ${ }^{2}$ \\ ${ }^{1}$ Graduate School of Economics, Kyoto University, Yoshida-Honmachi, Sakyo-ku, Kyoto 6068501, J.P. \\ ${ }^{2}$ Faculty of Economics, Kyoto University, J.P.
}

September 1, 2005

\begin{abstract}
The GARCH model is modified to capture the effect on volatilities of the consecutive number of days of positive or negative shocks. The new model is applied to the Shanghai Shcomp and Nikkei225 indices and found particularly useful in analyzing the Shcomp index. Similarly, the EGARCH model is extended along the same line as the GARCH model and is applied to the same sets of data. Stationarity of the new GARCH$(1,1)$ model is proved, and also derived is the asymptotic distribution of the quasimaximum likelihood estimator.
\end{abstract}

Key words: stock market; GARCH model; Volatility; spells of positive or negative shocks.

\section{Introduction}

Numerous ARCH class models have been proposed to estimate the conditional volatility of log returns including ARCH model (Engel, 1982) and GARCH model (Bollerslev, 1986). Both models are suitable for estimating the time-varying nature of the volatility in log returns of financial assets. The GARCH model is popular today, but it cannot capture the asymmetric effects of shocks on volatilities. The exponential GARCH (EGARCH, Nelson 1991) and the GJR-GARCH models (Glosten et al. 1993) were introduced to analyze the asymmetric effects. Subsequently proposed were the regime switching GARCH models such as the volatility-switching GARCH (Fornari and Mele, 1996, 1997) and the markov-switching GARCH (Kim 1993). The former allows all parameters in the conditional variance equation to depend on the sign of shocks, and, in the latter, the regime of the conditional variance equation is determined by unobservable Markov-processes. Baillie et al. (1996) and Bollerslev and Mikkelsen(1996) proposed the fractionally integrated GARCH and EGARCH, respectively, to analyze the persistent effect of shocks on volatilities. Further, every effort has been made to develop multivariate models including Bollerslev(1990), Engle and Kroner(1995). See Engel et al. (1990) and Kim and Tsurumi(2000) for empirical studies.

In this paper, the GARCH model is modified so that it can capture the effect of spells of positive or negative shocks on volatilities. The number of consecutive positive or negative shocks is incorporated into the GARCH model as a nonlinear

\footnotetext{
* Author for correspondence.
} 
coefficient of the ARCH term. Spells of positive or negative shocks are expected to have positive effect on volatilities as the size of returns does. On the other hand, the effect of these spells on returns can be either positive or negative. For instance, a positive return is not necessarily expected to follow from a spell of positive returns. Arbitrages may possibly follow if it does. The modified model is applied to Shanghai Shcomp and Nikkei225 indices, and the spell term is found significant only in the former.

Background of this modeling is found in the Chinese stock market which has different features from markets in other nations as Kang et al. (2002) and Chen and Shih(2002) studied. Shanghai Stock Exchange was established only in 1990 and is still immature. The A-share market is clustered by individual investors who have only a little knowledge on stock investments. As a result, they behave like noise traders whose decisions tend to be affected by rumors, by the rise and fall of share prices, and probably by spells of shocks. They also trade very frequently.

The following section presents the model. The new model is referred to as OG$\mathrm{ARCH}$ since our aim is to capture the overresponse in markets caused by spells of positive or negative shocks. Empirical studies in section 3 demonstrate that the $\operatorname{OGARCH}(1,1), \operatorname{OGARCH}(1,2), \operatorname{OGARCH}(1,3)$ models fit better than the corresponding GARCH model with respect to Shanghai Shcomp index. The EGARCH model is modified along the same line as OGARCH in section 3, and is applied to the same sets of data. Section 4 reports conclusions. The asymptotic properties of the new model are derived in the Appendix.

\section{Model}

The $\operatorname{OGARCH}(1,1)$ model is

$$
\begin{aligned}
y_{t} & =f\left(x_{t-1}\right)+\varepsilon_{t} \\
\varepsilon_{t} & =z_{t} \sqrt{h_{t}} \\
h_{t} & =\alpha_{0}+\alpha_{1} \exp \left(\phi \gamma_{t-1}\right) \varepsilon_{t-1}^{2}+\beta h_{t-1},
\end{aligned}
$$

where $y_{t}$ is the log return, $x_{t-1}$ is a vector of variables in the information set which includes all information upon time $t-1, f$ is any well behaved function, $\varepsilon_{t}$ is the shock or unpredictable return, $z_{t}$ is a white noise with mean zero and variances one, and $h_{t}$ is the conditional variance of $\varepsilon_{t}$. Signs of coefficients $a_{0}, a_{1}$, and $\beta$ are assumed to be positive, non-negative, and non-negative, respectively. Equations (1) and (3) are called the mean equation and the volatility equation, respectively.

$\gamma_{t-1}$ is the number of days in the spells of positive or negative shocks. It is a positive integer and formally defined as

$$
\gamma_{t-1} \equiv i, \text { if } \operatorname{sign}\left(\varepsilon_{t-1}\right)=\cdots=\operatorname{sign}\left(\varepsilon_{t-i}\right)=-\operatorname{sign}\left(\varepsilon_{t-(i+1)}\right) .
$$


It can take only two values, one or $\gamma_{t-2}+1$ which is larger than two, and is known at $t$. Define $p=P\left(z_{t}>0\right), q=1-p$, then the distribution of $\gamma_{t-1}$ in equation (3) is

$$
P\left(\gamma_{t-1}=\gamma\right)=q^{\gamma} p+q p^{\gamma}, \quad \gamma \geqq 1 .
$$

Since $\operatorname{sign}\left(\varepsilon_{t-i}\right)=\operatorname{sign}\left(z_{t-i}\right), \gamma_{t-1}$ is written as

$$
\gamma_{t-1}=g\left(\operatorname{sign}\left(z_{t-1}\right), \operatorname{sign}\left(z_{t-2}\right), \cdots\right) .
$$

The exponential term is always positive, and the $\phi$ coefficient is expected to be positive so that $h_{t}$ increases together with $\gamma_{t-1}$. The standard $\operatorname{GARCH}(1,1)$ model follows if $\phi$ is zero. The asymptotic properties of the OGARCH model are proved in the Appendix.

\section{Empirical analysis}

The OGARCH model is applied to the log returns of daily closing prices of Nikkei225 and Shcomp which are price indices of 225 stocks of Tokyo Stock Exchange and of Shanghai Stock Exchange, respectively.

Chinese and Japanese stock markets have different features. Stocks have been traded since 1860's, but stock market was closed in 1949 in the Mainland China. The new Shanghai Stock Exchange was re-opened in 1990 and has developed to a biggest exchange. The total market capitalization grew from 1.2 billion RMB in 1990 to 2.6 trillion RMB in 2004. On the other hand, Tokyo Stock Exchange was established in 1949. The total market capitalization decreased from 449.4 trillion yen to 385.7 trillion yen in the same period.

The Chinese market has 1.5 trillion RMB of uncirculated state-held shares that account $57.6 \%$ of the total market capitalization. This does not exist in the Japanese market. Moreover, the Chinese markets have two strictly segmented A and B-share sections. The A-shares are denominated by the Chinese currency and issued almost only to and traded almost only by domestic investors. On the other hand, the Bshares are denominated by the US or Hong Kong dollars and issued almost only to and traded almost only by foreign investors (Kang et al. 2002). The A-share is the larger part which is affected immensely by individual investors.

Shanghai Stock market grew up rapidly. Brands were replaced frequently and indices were unstable in the early years. Such unstable period is avoided in this study. The IT bubble period is also avoided which may be roughly from 1996 though 2000. The sample period used in this study extends from 4 January, 2001 to 15 August, 2005 for both Shcomp and Nikkei225 indices. 
Table I. Summary Statistics of Data

\begin{tabular}{lcccr}
\hline \multicolumn{1}{c}{$\mathrm{T}$} & Mean & Variance & Skewness & Kurtosis \\
\hline Shcomp & & & $(01 / 04 / 2001-15 / 08 / 2005)$ \\
1104 & -0.05 & 1.94 & 1.13 & 11.13 \\
Nikkei & & \multicolumn{3}{c}{$(01 / 04 / 2001-15 / 08 / 2005)$} \\
1135 & -0.01 & 2.12 & -0.04 & 4.55 \\
\hline
\end{tabular}

\subsection{THE SUMMARY STATISTICS ${ }^{1}$}

The summary statistics of the two log return $y_{t}$ series are tabulated in Table I. The Shcomp kurtosis doubles that of Nikkei which means Shcomp is more fluctuant than Nikkei. Figure I tabulates the correlograms of the two series which are mostly insignificant except for the marginally significant seventh Shcomp and forth Nikkei autocorrelations. Ljung-Box statistics (P-values) with the first twelve serial correlations for Shcomp and Nikkei are 12.31(0.42) and 11.44(0.49), respectively. Supported also by AIC and BIC, any lag of the log return is not included in our mean equation in the following studies.
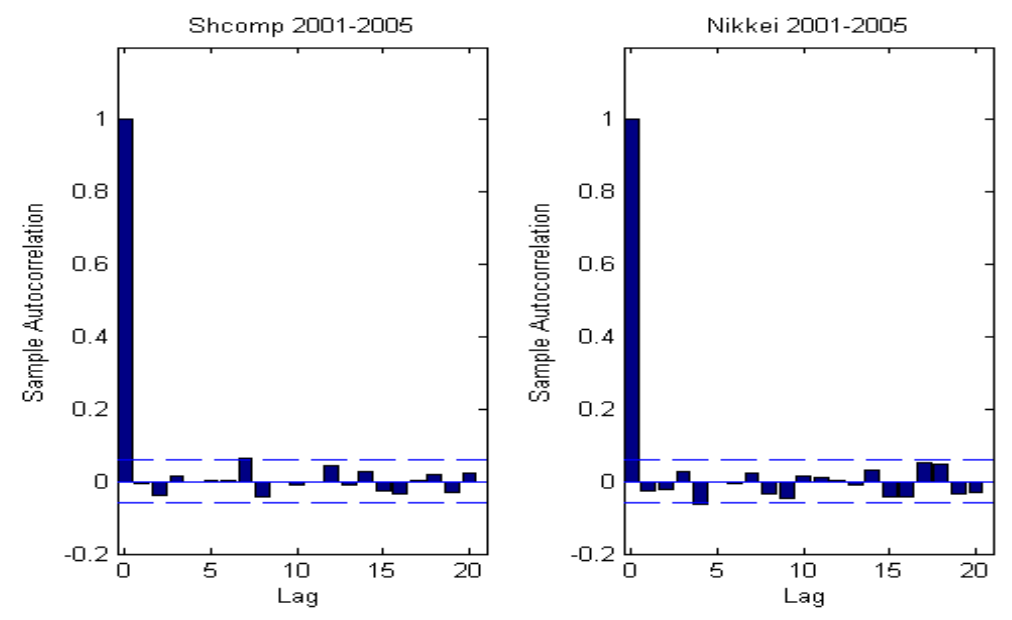

Figure I. Correlogram of Log return.

\footnotetext{
${ }^{1} 3.1$ and 3.2 are included following the two referees' comments.
} 
Table II. Day-of-the-week Effect

\begin{tabular}{lrrrrr}
\hline Variable & \multicolumn{1}{c}{1} & \multicolumn{1}{c}{$d 1$} & \multicolumn{1}{c}{$d 2$} & \multicolumn{1}{c}{$d 3$} & \multicolumn{1}{c}{$d 4$} \\
\hline Shcomp & & & & & \\
Coefficient & -.10 & -.10 & $.29^{*}$ & .10 & -.03 \\
Std. Error & .10 & .13 & .13 & .13 & .13 \\
Nikkei & & & & & \\
Coefficient & .10 & -.22 & -.10 & -.10 & -.05 \\
Std. Error & .10 & .14 & .14 & .14 & .14 \\
\hline
\end{tabular}

* indicates significant coefficient at $5 \%$.

Table III. Seasonality Test

\begin{tabular}{lrrrrrrrrrrrr}
\hline Variable & 1 & $\mathrm{~m} 1$ & $\mathrm{~m} 2$ & $\mathrm{~m} 3$ & $\mathrm{~m} 4$ & $\mathrm{~m} 5$ & $\mathrm{~m} 6$ & $\mathrm{~m} 7$ & $\mathrm{~m} 8$ & $\mathrm{~m} 9$ & $\mathrm{~m} 10$ & $\mathrm{~m} 11$ \\
\hline Shcomp & & & & & & & & & & & & \\
Coeff & .02 & .08 & -.34 & .09 & -.14 & -.37 & -.05 & .32 & -.01 & .24 & -.23 & .00 \\
SE & .24 & .39 & .31 & .26 & .21 & .32 & .28 & .37 & .10 & .16 & .16 & .23 \\
Nikkei & & & & & & & & & & & & \\
Coeff & .08 & .12 & .13 & .12 & .11 & .11 & -.04 & .04 & -.12 & .08 & .16 & .11 \\
SE & .15 & .21 & .21 & .21 & .21 & .21 & .21 & .21 & .22 & .22 & .22 & .22 \\
\hline
\end{tabular}

\subsection{PRELIMINARY TESTS}

Examined in this sub-section are day-of-the-week effects, seasonality, serial correlations in $y_{t}^{2}$, and an alternative specification of the mean equation.

Firstly, following Engle and $\mathrm{Ng}$ (1993), log return $y_{t}$ is regressed on a constant and four dummy variables $d 1$ to $d 4$ to examine day-of-the-week effects. Results are reported in Table II, and the Tuesday coefficient $d 2$ is found significant in the Shcomp series for this particular sample period. The original series is replaced by residuals from regressing $y_{t}$ on a constant and $d 2$ in the following estimation for shcomp.

Secondly, $y_{t}$ is regressed on a constant and eleven dummy variables $m 1$ to $m 11$ to examine the seasonality of the two series. Observing the Table III, we can find any seasonality in neither series.

Thirdly examined are the serial correlations in $y_{t}^{2}$. Ljung-Box statistics (P-values) with the first twelve serial correlations for Shcomp and Nikkei are 29.03(0.00) and 185.64(0.00), respectively. Time-varying volatility is in this series.

After trying various models with various lags in $y_{t}$, comparing AIC and SBIC of these models, and testing significance of coefficients, the mean equation chosen for 
both Shcomp and Nikkei is

$$
y_{t}=\mu+\varepsilon_{t} .
$$

Mean equations with $\gamma_{t-1}$ as an explanatory variable such as

$$
y_{t}=f\left(x_{t-1}\right)+\psi \gamma_{t-1}+\varepsilon_{t},
$$

were also estimated, but $\gamma_{t-1}$ turned out to be always insignificant. (The volatility equation must be estimated together. For example, $\psi$ is 0.03 with t-ratio 1.18 in the equation $y_{t}=\mu+\psi \gamma_{t-1}+\varepsilon_{t}$ in the Shcomp $\operatorname{OGARCH}(1,1)$.)

\subsection{OGARCH ESTIMATION AND TESTS}

The $\operatorname{OGARCH}(1,1)$ and $\operatorname{GARCH}(1,1)$ models are estimated by the quasi-maximum likelihood (QML) method assuming that $z_{t}$ is a standard normal random variable. The quasi $\log$ likelihood function is

$$
L_{T}(\theta)=\sum_{t=1}^{T}-\frac{1}{2} \log 2 \pi-\frac{1}{2} \log h_{t}-\frac{\varepsilon_{t}^{2}}{2 h_{t}}
$$

where $T$ is the sample size. The QML method is usually applied to the ARCH class models since the distribution of disturbance term is unknown but known to be nonnormal.

Table IV presents the summary of estimation. In Shcomp case, all OGARCH coefficients are significant including $\phi$, and the quasi log likelihood of OGARCH increased by 9.0 from that of GARCH. However, $\phi$ is not significant in Nikkei case, and other coefficients and the quasi log likelihood values are similar between the two models. The Wald test ${ }^{2}$ also supports the same result on the $\phi$ coefficient.

The same models are applied to the A and B share indices of Shanghai market. ${ }^{3}$ Estimation results on A and B share indices are similar to those of Shcomp, and the coefficient $\phi$ is positive and significant in both series. This follows since, firstly, the $\log$ return of the A share index is very similar to that of Shcomp since they have similar components. Secondly, the fluctuations of A and B shares are similar. In fact, the fluctuation of B shares is dominated by A shares since every B share which is traded in foreign currency is a counterpart of an A share but with a small amount. The A share accounts for $90 \%$ of all shares in Shanghai market, and there are only 54 B share brands in 2002.

\footnotetext{
${ }^{2}$ According to Weiss (1986), the LR test statistic for ARCH class model estimated by QMLE is not distributed as $\chi^{2}$. However, the Wald test statistic is distributed as $\chi^{2}$. It is only a squared t-ratio in our estimation of $\phi$ coefficient.

${ }^{3}$ Following estimation was suggestd by a referee.
} 
Table IV. OGARCH and GARCH Estimation

\begin{tabular}{|c|c|c|c|c|c|c|}
\hline Parameter & $\mu$ & $\alpha_{0}$ & $\alpha_{1}$ & $\beta$ & $\phi$ & $\log L$ \\
\hline \multicolumn{7}{|c|}{ Shcomp } \\
\hline \multicolumn{7}{|l|}{ GARCH } \\
\hline Coeff & .029 & $.138^{* *}$ & $.179^{* *}$ & $.770^{* *}$ & & -1861.3 \\
\hline SE & .047 & .041 & .063 & .052 & & \\
\hline \multicolumn{7}{|l|}{ OGARCH } \\
\hline Coeff & $.110^{* *}$ & $.108^{* *}$ & $.087^{*}$ & $.794^{* *}$ & $.268^{* *}$ & -1852.3 \\
\hline SE & .042 & .030 & .038 & .044 & .068 & \\
\hline \multicolumn{7}{|c|}{ A share } \\
\hline Coeff & $.085^{*}$ & $.101^{* *}$ & $.072^{*}$ & $.809^{* *}$ & $.309^{* *}$ & \\
\hline SE & .043 & .028 & .032 & .041 & .069 & -1839.1 \\
\hline \multicolumn{7}{|c|}{ B share } \\
\hline Coeff & .058 & $.202^{* *}$ & $.085^{*}$ & $.807^{* *}$ & $.213^{* *}$ & -2194.0 \\
\hline SE & .047 & .066 & .034 & .038 & .076 & \\
\hline \multicolumn{7}{|c|}{ Nikkei } \\
\hline \multicolumn{7}{|l|}{ GARCH } \\
\hline Coeff & .037 & .151 & $.087^{* *}$ & $.910^{* *}$ & & -1954.3 \\
\hline SE & .035 & .010 & .022 & .022 & & \\
\hline \multicolumn{7}{|l|}{ OGARCH } \\
\hline Coeff & .036 & .015 & $.073^{* *}$ & $.909^{* *}$ & .087 & -1953.6 \\
\hline SE & .039 & .049 & .028 & .030 & .153 & \\
\hline
\end{tabular}

Note: The sample periods of the A and B shares are the same as Shcomp. The numbers of observations are 1103, 1097, 1091, and 1135 for Shcomp, A share, B share, and Nikkei, respectively. ${ }^{*}$ and $* *$ indicate significant coefficient at $5 \%$ and $1 \%$, respectively.

The OGARCH $(1,1)$ model captures the effects of spells of shocks on the conditional volatility in the Shcomp case successfully. News impact curves are used to visualize this property in the next subsection.

\subsection{NEWS IMPACT CURVES}

The impact curve functions are

$$
h_{t}=\alpha_{0}+\beta \sigma^{2}+\alpha_{1} \varepsilon_{t-1}^{2}
$$

for $\operatorname{GARCH}(1,1)$, and

$$
h_{t}=\alpha_{0}+\alpha_{1} \exp \left(\phi \gamma_{t-1}\right) \varepsilon_{t-1}^{2}+\beta \sigma^{2}
$$

for $\operatorname{OGARCH}(1,1)$ where $\sigma$ is the unconditional standard deviation. The impact curves are plotted in Figure II using estimates in Table IV. 


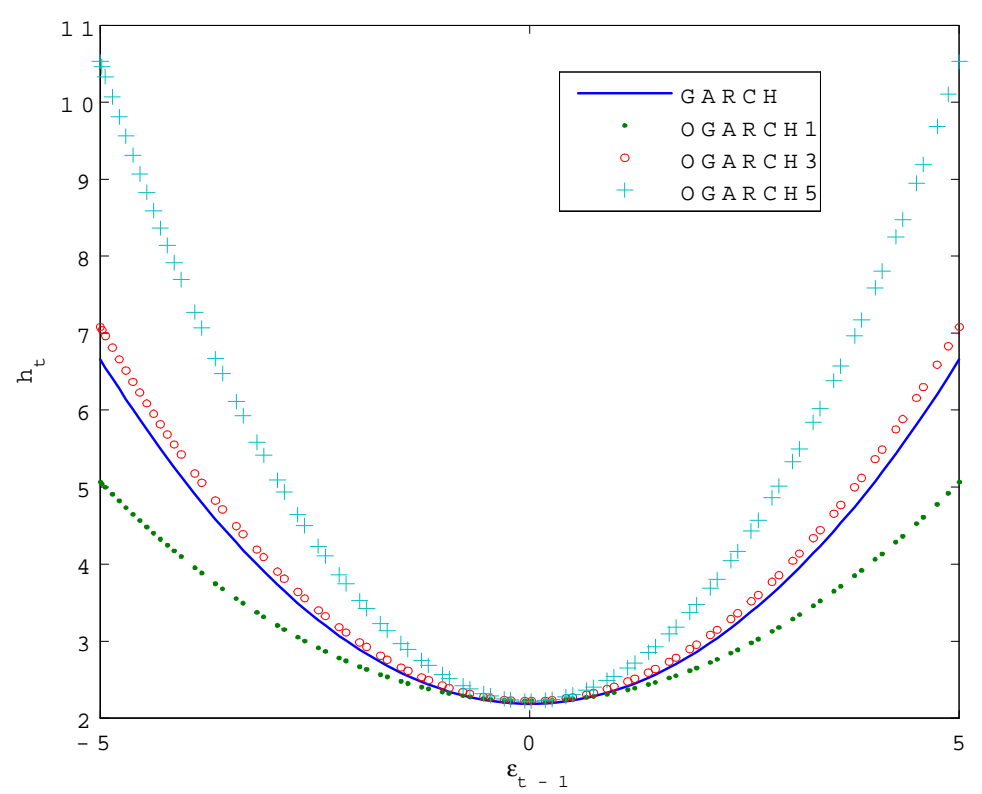

Figure II. Impact Curves. OGARCH1, OGARCH3 and OGARCH5 denote OGARCH with $\gamma_{t-1}=1,3$ and 5 , respectively.

The news impact curve of $\operatorname{OGARCH}(1,1)$ is affected by the values of $\gamma_{t-1}$. It is close to that of $\operatorname{GARCH}(1,1)$ when $\gamma_{t-1}=3$, and they deviate away from $\operatorname{GARCH}(1,1)$ for other values. The larger are the values of $\gamma_{t-1}$ (as same the longer are the spells), the greater are the impacts on $h_{t}$ in the $\operatorname{OGARCH}(1,1)$ model. Effects of the number of days in spells are obvious in the Shcomp case.

\subsection{DIAGNOSTIC TESTS}

Further diagnostic tests are applied to examine whether asymmetric effects should be taken into account in the two models. Diagnostic tests include the sign bias, negative size bias, positive size bias, and the joint tests. See Engle and Ng 1993 for details of these tests. Results are reported in Table V. The negative bias tests of the two models are significant in Shcomp, and the negative bias tests and joint tests of the two models are significant in Nikkei. Asymmetric analyses can be useful in this series for both Shcomp and Nikkei.

Ljung-Box statistics for the first twelve serial correlations in the normalized residuals $\nu_{t} \equiv \varepsilon_{t} / \sqrt{h_{t}}$ and $\nu_{t}^{2}$ are calculated for the two series, but they turn out to be 
Table V. Diagnostic Test

\begin{tabular}{lrrrrrr}
\hline & $\mathrm{SB}$ & \multicolumn{1}{c}{$\mathrm{SB}$} & \multicolumn{1}{c}{$+\mathrm{SB}$} & \multicolumn{1}{c}{$\mathrm{JT}$} & $\mathrm{LB}$ of $\nu_{t}$ & $\mathrm{LB}$ of $\nu_{t}^{2}$ \\
\hline & & \multicolumn{5}{c}{ Shcomp } \\
GARCH & 1.66 & 2.16 & -1.34 & 6.77 & 5.04 & 4.62 \\
P-value & .097 & $.031^{*}$ & .181 & .080 & .929 & .948 \\
OGARCH & 1.67 & $2.61^{* *}$ & -1.01 & 7.55 & 6.63 & 5.69 \\
P-value & .095 & .009 & .311 & .056 & .828 & .893 \\
& & & & & & \\
GARCH & 1.04 & $2.13^{*}$ & -1.95 & $9.12^{*}$ & 6.62 & 12.25 \\
P-value & .298 & .033 & .051 & .028 & .829 & .345 \\
OGARCH & 1.08 & $2.23^{*}$ & -1.95 & $9.40^{*}$ & 6.26 & 11.80 \\
P-value & .280 & .026 & .051 & .025 & .855 & .379 \\
\hline
\end{tabular}

Note: * indicates significant results at $5 \%$. Sign bias, negative size bias, positive size bias, joint tests and Ljung-Box test are denoted $\mathrm{SB},-\mathrm{SB},+\mathrm{SB}, \mathrm{JT}$ and $\mathrm{LB}$, respectively, and their null distributions are $t, t, t, \chi^{2}$ and $\chi^{2}$.

insignificant. It may be concluded that the time-varying properties of volatilities are successfully captured by the two models.

\subsection{HIGHER ORDER GARCH AND OGARCH}

The OGARCH model should be compared with higher order GARCH models. ${ }^{4}$ The mean equation is kept the same, and the $\operatorname{GARCH}(1,2)$ and $\operatorname{GARCH}(1,3)$ models are equations (7) and (8), respectively.

$$
\begin{aligned}
& h_{t}=\alpha_{0}+\alpha_{1} \varepsilon_{t-1}^{2}+\alpha_{2} \varepsilon_{t-2}^{2}+\beta h_{t-1} \\
& h_{t}=\alpha_{0}+\alpha_{1} \varepsilon_{t-1}^{2}+\alpha_{2} \varepsilon_{t-2}^{2}+\alpha_{3} \varepsilon_{t-3}^{2}+\beta h_{t-1}
\end{aligned}
$$

These two and corresponding OGARCH models are estimated for Shcomp. The estimation results are tabulated in Table VI. $\alpha_{3}$ is insignificant in the $\operatorname{GARCH}(1,3)$ model, and $\alpha_{2}$ is insignificant in the $\operatorname{GARCH}(1,2)$ model. Therefore, among the GARCH models, the GARCH $(1,1)$ model where $\alpha_{1}$ is significant could be accepted as most desirable if a model search is started from GARCH(1,3).

It is of interest to compare $\operatorname{OGARCH}(1,1)$ and $\operatorname{GARCH}(1,2)$ since the spell effect can be absorbed by extending lag windows of the GARCH model. However, these models are not nested with each other and not comparable directly. Since $\operatorname{GARCH}(1,2)$ is nested in $\operatorname{OGARCH}(1,2)$, the $\operatorname{OGARCH}(1,2)$ model is estimated for comparison. $\phi$ turns out to be significant, the $\operatorname{OGARCH}(1,2)$ model is found to be more desirable than the $\operatorname{GARCH}(1,2)$ model. Increment in the log likelihood value is

\footnotetext{
${ }^{4}$ This study was also suggested by a referee.
} 
Table VI. Higher Order Models

\begin{tabular}{lllllllll}
\hline Parameter & $\mu$ & $\alpha_{0}$ & $\alpha_{1}$ & $\alpha_{2}$ & $\alpha_{3}$ & $\beta$ & $\phi$ & $\log L$ \\
\hline Order & & & & & $(1,2)$ & & & \\
GARCH & .028 & $.263^{* *}$ & .086 & .213 & & $.604^{* *}$ & & -1853.5 \\
OGARCH & .052 & $.333^{* *}$ & .048 & $.138^{*}$ & & $.550^{* *}$ & $.237^{*}$ & -1843.1 \\
& & & & & & & & \\
Order & & & & & $(1,3)$ & & & \\
GARCH & .028 & $.551^{* *}$ & .091 & $.257^{*}$ & .130 & $.304^{* *}$ & & -1852.2 \\
OGARCH & .006 & $.624^{* *}$ & .049 & $.113^{* *}$ & .080 & $.277^{*}$ & $.275^{*}$ & -1842.2 \\
\hline
\end{tabular}

Note: $*$ and $* *$ indicate significant coefficient at $5 \%$ and $1 \%$, respectively. The number of observations is dependent upon the lag orders. SEs are omitted to save space.

not small, either. Similar results are found in $\operatorname{OGARCH}(1,3)$. The spell effect can be absorbed by lag windows, but the spell term is found useful in analyzing this series.

\subsection{EGARCH MODEL}

The extension of EGARCH model referred as OEGARCH model is

$$
\log \left(h_{t}\right)=\alpha_{0}+\exp \left(\phi \gamma_{t-1}\right)\left[\alpha_{1} z_{t-1}+\kappa\left(\left|z_{t-1}\right|-E\left(\left|z_{t-1}\right|\right)\right)\right]+\beta \log \left(h_{t-1}\right) .
$$

The $\operatorname{OEGARCH}(1,1)$ model reduces into $\operatorname{EGARCH}(1,1)$ when $\phi$ is zero.

Asymptotic properties of OEGARCH $(1,1)$ are not derived, but the QML method is applied to the same sets of data. Results are tabulated in Table VII. The $\phi$ coefficients of Shcomp and Nikkei are both insignificant, and other coefficients of the EGARCH and OEGARCH are similar. The OEGARCH model does not perform better than the EGARCH model for both series. Note that the GARCH and the EGARCH models are neither nested with each other, nor comparable by the quasi log likelihood values, either.

\section{Conclusion}

The GARCH model is modified to capture the effect on volatilities of the consecutive number of days of positive or negative shocks. Spells of positive or negative shocks 
Table VII. OEGARCH and EGARCH Estimation

\begin{tabular}{lccccccc}
\hline Parameter & $\mu$ & $\alpha_{0}$ & $\alpha_{1}$ & $\beta$ & $\kappa$ & $\phi$ & $\log L$ \\
\hline EGARCH & & \multicolumn{7}{c}{ Shcomp } \\
Coeff & -.029 & $.029^{*}$ & $-.119^{*}$ & $.968^{* *}$ & $.188^{* *}$ & & -1828.4 \\
SE & .036 & .010 & .039 & .014 & .037 & & \\
OEGARCH & & & & & & & \\
Coeff & -.042 & $.031^{* *}$ & $-.114^{*}$ & $.966^{* *}$ & $.181^{* *}$ & .022 & -1828.1 \\
SE & .042 & .030 & .038 & .044 & .068 & .071 & \\
& & & & & & & \\
EGARCH & & & Nikkei & & & \\
Coeff & .000 & .012 & $-.062^{*}$ & $.983^{* *}$ & $.172^{* *}$ & & -1948.0 \\
SE & .035 & .008 & .027 & .082 & .040 & & \\
OEGARCH & & & & & & & \\
Coeff & .000 & .012 & $-.060^{*}$ & $.983^{* *}$ & $.167^{* *}$ & .013 & -1947.9 \\
SE & .035 & .008 & .028 & .008 & .051 & .088 & \\
\hline
\end{tabular}

Note: The numbers of observations are 1103 and 1135 for Shcomp and Nikkei respectively. ${ }^{*}$ and $* *$ indicate significant coefficient at $5 \%$ and $1 \%$, respectively.

are expected to have positive effect on volatilities. The new model nomenclatured OGARCH is applied to the Shanghai Shcomp and Nikkei225 indices and found particularly useful in analyzing the Shcomp index. Similarly, the EGARCH model is extended to the OEGARCH model along the same line and is applied to the same sets of data.

The OGARCH model describes the dynamic behavior of conditional volatility better than the GARCH model in analyzing the Shcomp index series. This result holds for three sets of ARCH lag orders; $(1,1),(1,2)$, and $(1,3)$. The new model does not perform well in the Nikkei225 index series. This may imply that the Shanghai market has different features from the Tokyo market as Kang et al. (2002) and Chen and Shih (2002) have reported.

Only fifteen years have passed since the Shanghai market was established. Investors might be still immature, and they might have been influenced by spells of positive or negative shocks. Conditional volatilities but not the mean returns might have increased as investors face consecutive positive or negative shocks. Theoretical justification is needed to explain this phenomenon.

The OGARCH model is simple and can be extended into other directions. For instance, the spell term $\exp \left(\phi \gamma_{t-1}\right)$ can be replaced by a function of some other variables. It is also of interest to apply this model to other series, particularly, to market indices of newly established stock exchanges in the world. 


\section{Appendix}

The strict stationarity conditions are derived and the asymptotic theory of $\operatorname{OGARCH}(1,1)$ is established in this appendix. The asymptotic distribution is necessary for applying Wald tests.

\subsection{STATIONARITY}

Define

$$
\eta\left(z_{t-1}, \gamma_{t-1}\right) \equiv \alpha_{1} z_{t-1}^{2} \exp \left(\phi \gamma_{t-1}\right)+\beta,
$$

then a new representation of equation (3) is

$$
h_{t}=\alpha_{0}+\eta\left(z_{t-1}, \gamma_{t-1}\right) h_{t-1} .
$$

A proposition follow.

PROPOSITION 1. Let $E\left[\log \eta\left(z_{t-1}, \gamma_{t-1}\right)\right]$ be nonzero. There exists a strict-stationary solution iff $E\left[\log \eta\left(z_{t-1}, \gamma_{t-1}\right)\right]<0$. If there exists a solution, it is unique.

Proof. Proof is based on Zakoian (1994).

(i) Sufficiency.

Suppose $E\left[\log \eta\left(z_{t-1}, \gamma_{t-1}\right)\right]=\delta<0$. Repeated substitutions in equation (10) yield

$$
\begin{aligned}
h_{t}= & \eta\left(z_{t-1}, \gamma_{t-1}\right) \eta\left(z_{t-2}, \gamma_{t-2}\right) \cdots \eta\left(z_{t-p}, \gamma_{t-p}\right) h_{t-p} \\
& +\alpha_{0} \sum_{i=0}^{p-1} \eta\left(z_{t-1}, \gamma_{t-1}\right) \eta\left(z_{t-2}, \gamma_{t-2}\right) \cdots \eta\left(z_{t-i}, \gamma_{t-i}\right),
\end{aligned}
$$

with the convention that $\eta\left(z_{t-1}, \gamma_{t-1}\right) \cdots \eta\left(z_{t-i}, \gamma_{t-i}\right)=1$ for $i=0$.

Since $\gamma_{t}$ is a function of $\left(z_{t}, z_{t-1}, \cdots\right), \log \eta\left(z_{t}, \gamma_{t}\right)$ is a function of $\left(z_{t}, z_{t-1}, \cdots\right)$ which are i.i.d., then $\left\{\log \eta\left(z_{t}, \gamma_{t}\right)\right\}$ is a strictly stationary ergodic sequence by Theorem 3.5.8 of Stout (1974). Moreover, by the Ergodic theorem (Stout, 1974),

$$
\lim _{i \longrightarrow \infty} \frac{1}{i} \sum_{k=0}^{i-1} \log \eta\left(z_{t-1-k}, \gamma_{t-1-k}\right)=\delta \quad \text { a.s. }
$$

Since $\delta<0$, it follows that

$$
\lim _{i \longrightarrow \infty}\left[\eta\left(z_{t-1}, \gamma_{t-1}\right) \eta\left(z_{t-2}, \gamma_{t-2}\right) \cdots \eta\left(z_{t-i}, \gamma_{t-i}\right)\right]^{\frac{1}{i}}=e^{\delta}<1 \quad \text { a.s. }
$$

Hence, $\xi_{t}$ exists which satisfies

$$
\xi_{t}=\lim _{p \longrightarrow \infty} \sum_{i=0}^{p} \eta\left(z_{t-1}, \gamma_{t-1}\right) \cdots \eta\left(z_{t-i}, \gamma_{t-i}\right) \alpha_{0} \quad \text { a.s. }
$$


by the Cauchy root test.

The $\xi_{t}$ process is also strictly stationary because it is a function of $\left\{\log \eta\left(z_{t}, \gamma_{t}\right)\right\}$. Define $\varepsilon_{t}=\xi_{t} z_{t}$ for all $t$, then, the $\varepsilon_{t}$ process is a solution of equations (2) and (3). It is apparent that $\varepsilon_{t}$ is strictly stationary and ergodic.

(ii) Necessity.

Suppose that $E\left[\log \eta\left(z_{t-1}, \gamma_{t-1}\right)\right]=\delta>0$.

$$
h_{t} \geq \sum_{i=0}^{p-1} \eta\left(z_{t-1}, \gamma_{t-1}\right) \eta\left(z_{t-2}, \gamma_{t-2}\right) \cdots \eta\left(z_{t-i}, \gamma_{t-i}\right) \alpha_{0}
$$

for all $p$ by equation (11). This series diverges as $e^{\delta}>1$ by equation (13); thereby, $h_{t}$ also diverges. Hence, the condition $E\left[\log \eta\left(z_{t-1}, \gamma_{t-1}\right)\right]=\delta<0$ is necessary.

(iii) Uniqueness.

When $E\left[\log \eta\left(z_{t-1}, \gamma_{t-1}\right)\right]<0, \eta\left(z_{t-1}, \gamma_{t-1}\right) \cdots \eta\left(z_{t-i}, \gamma_{t-i}\right)$ in equation (11) converges to zero by using equation (13). Therefore, $\xi_{t}$ and $\varepsilon_{t}$ processes must be unique.

\subsection{CONSISTENCY AND ASYMPTOTIC NORMALITY}

Conditions for the consistency and the asymptotic normality of the QMLE (QML estimate) are summarized as a proposition. They are derived by modifying the proof of Weiss (1986) for the ARCH model.

PROPOSITION 2. $\theta_{0} \equiv\left(\alpha_{00} \alpha_{10} \beta_{0} \phi_{0}\right)$ is the true parameter vector of $\theta \equiv\left(\alpha_{0}\right.$ $\left.\alpha_{1} \beta \phi\right), \varepsilon_{t 0}$ and $h_{t 0}$ are the true variables of $\varepsilon_{t}$ and $h_{t}$, and

(a) $\varepsilon_{t 0}$ satisfies strict stationarity condition and is ergodic, $E\left(\varepsilon_{t 0}^{8}\right)<\infty$, the distribution conditioned on $I_{t-1}$ of $\varepsilon_{t 0}$ is continuous where $I_{t-1}$ is the information set containing information on the $\varepsilon_{t 0}$ process up to and including $t-1$.

(b) $h_{t}>0, \alpha_{0}>0, \alpha_{1} \geq 0, \beta \geq 0$,

(c) The parameter space $\Theta$ is a compact subset of the Euclidean space, $\theta_{0} \in \Theta$,

(d) $\phi<(1 / 4) \log 2 .^{5}$

Under these conditions, the QMLE $\hat{\theta}_{T}$ of the OGARCH $(1,1)$ model converges to $\theta_{0}$ in probability. If $\operatorname{det} B_{0}>0$, then

$$
\sqrt{T}\left(\hat{\theta}_{t}-\theta_{0}\right) \stackrel{d}{\rightarrow} N\left(0, A_{0}^{-1} B_{0} A_{0}^{-1}\right),
$$

where

$$
A_{0} \equiv-E\left[\nabla^{2} L_{T}\left(\theta_{0}\right)\right]=\frac{1}{2} E\left(h_{t 0}^{-2} \nabla h_{t} \nabla^{\prime} h_{t}\right)+E\left(h_{t 0}^{-1} \nabla \varepsilon_{t} \nabla^{\prime} \varepsilon_{t}\right)
$$

\footnotetext{
${ }^{5}$ The upper bound of $\phi$ can be larger than $(1 / 4) \log 2$ if higher-order moments of $\varepsilon_{0 t}$ exist. Proof follows by using the Hölder instead of the Cauchy-Schwartz inequality.
} 
and

$$
B_{0} \equiv E\left[T \nabla L_{T}\left(\theta_{0}\right) \nabla^{\prime} L_{T}\left(\theta_{0}\right)\right] .
$$

The consistent estimators of $A_{0}$ and $B_{0}$ are

$$
\hat{A}_{T}=\frac{1}{2 T} \sum h_{t}^{-2} \nabla h_{t} \nabla^{\prime} h_{t}+\left.\frac{1}{T} \sum h_{t}^{-1} \nabla \varepsilon_{t} \nabla^{\prime} \varepsilon_{t}\right|_{\theta=\hat{\theta}_{T}},
$$

and

$$
\hat{B}_{T}=\left.\frac{1}{T} \sum \nabla l_{t} \nabla^{\prime} l_{t}\right|_{\theta=\hat{\theta}_{T}},
$$

respectively.

Proof. Next lemma is a modification of the lemma (3.2) of Weiss (1986), and the rest is easily proved following Weiss (1986).

LEMMA 3. Under the conditions of proposition 2, there exists $M<\infty$ such that

$$
E\left[\frac{\partial h_{t}}{\partial \nu} \frac{\partial h_{t}}{\partial \nu^{\prime}}\right]<M
$$

for all $\theta \in \Theta$. Moreover,

$$
\operatorname{det} E\left[\frac{\partial h_{t}}{\partial \nu} \frac{\partial h_{t}}{\partial \nu^{\prime}}\right]>0
$$

where $\nu=\left[\begin{array}{lll}\alpha_{0} & \alpha_{1} & \beta\end{array}\right]^{\prime}$.

Proof.

By the conditions, $\beta<1$. Differentiating the equation (3),

$$
\begin{aligned}
\frac{\partial h_{t}}{\partial \alpha_{0}} & =1+\sum_{i=1}^{\infty} \beta^{i}=\frac{1}{1-\beta}<\infty \\
\frac{\partial h_{t}}{\partial \alpha_{1}} & =\sum_{j=0}^{\infty}\left(\varepsilon_{t-j-1}^{2} \beta^{j} \exp \left(\phi \gamma_{t-j-1}\right)\right), \\
\frac{\partial h_{t}}{\partial \beta} & =\sum_{l=1}^{\infty} l \beta^{l-1}\left[\alpha_{0}+\alpha_{1} \varepsilon_{t-l-1}^{2} \exp \left(\phi \gamma_{t-l-1}\right)\right] \\
\frac{\partial h_{t}}{\partial \phi} & =\sum_{i=0}^{\infty} \beta^{i} \alpha_{1} \gamma_{t-i-1} \exp \left(\phi \gamma_{t-i-1}\right) \varepsilon_{t-i-1}^{2} .
\end{aligned}
$$


The second moment of $\frac{\partial h_{t}}{\partial \alpha_{1}}$ is

$$
\begin{aligned}
E\left[\left(\frac{\partial h_{t}}{\partial \alpha_{1}}\right)^{2}\right] & =E\left[\left(\sum_{j=0}^{\infty}\left(\varepsilon_{t-j-1}^{2} \beta^{j} \exp \left(\phi \gamma_{t-j-1}\right)\right)\right)^{2}\right] \\
& =\sum_{i=0}^{\infty} \sum_{j=0}^{\infty} \beta^{i+j} E\left[\left(\varepsilon_{t-i-1}^{2} \exp \left(\phi \gamma_{t-i-1}\right)\right)\left(\varepsilon_{t-j-1}^{2} \exp \left(\phi \gamma_{t-j-1}\right)\right)\right] \\
& \leq \sum_{i=0}^{\infty} \sum_{j=0}^{\infty} \beta^{i+j}\left[E\left[\varepsilon_{t-i-1}^{4} \exp \left(2 \phi \gamma_{t-i-1}\right)\right] E\left[\varepsilon_{t-j-1}^{4} \exp \left(2 \phi \gamma_{t-j-1}\right)\right]^{\frac{1}{2}}\right. \\
& \leq\left(E \exp \left(4 \phi \gamma_{t}\right)\right)^{\frac{1}{2}}\left(E \varepsilon_{t}^{8}\right)^{\frac{1}{2}} \frac{1}{(1-\beta)^{2}}
\end{aligned}
$$

where the second quality is by the monotone convergence theorem, and the CauchySchwartz inequality is repeatedly used. Further, we have

$$
\begin{aligned}
E\left[\exp \left(4 \phi \gamma_{t}\right)\right] & =\sum_{i=1}^{\infty}\left(q^{i} p+q p^{i}\right) \exp (4 i \phi) \\
& =\sum_{i=1}^{\infty}\left(C_{i}+D_{i}\right)
\end{aligned}
$$

and,

$$
\begin{aligned}
& \frac{C_{i+1}}{C_{i}}=\frac{q^{i+1} p \exp (4(i+1) \phi)}{(q)^{i} p \exp (4 i \phi)}=q \exp (4 \phi)<1 \\
& \frac{D_{i+1}}{D_{i}}=\frac{p^{i+1} q \exp (4(i+1) \phi)}{p^{i} q \exp (4 i \phi)}=p \exp (4 \phi)<1
\end{aligned}
$$

where the assumption (d), and $p=q=1 / 2$ are used. The latter follows since the distribution of $z_{t}$ is symmetric. Then (15) is bounded since (16) is finite by d'Alembert ratio test, $\Theta$ is compact, $E\left(\varepsilon_{t}^{8}\right)=E\left(\varepsilon_{t 0}+\mu_{0}-\mu\right)^{8}<\infty$. Boundedness of the second order moments of the first derivatives can be proved similarly. Therefore the first part of the lemma is proved.

If the second part is not satisfied, there exists $\lambda \neq 0$ such that $E\left[\lambda^{\prime} \frac{\partial h_{t}}{\partial \nu} \frac{\partial h_{t}}{\partial \nu^{\prime}} \lambda\right]=0$. This implies $\lambda^{\prime} \frac{\partial h_{t}}{\partial \nu}=0$, a.s. for all $t$. Substituting $\varepsilon_{t}=\varepsilon_{t 0}+\mu_{0}-\mu$ into $\lambda^{\prime} \frac{\partial h_{t}}{\partial \nu}=$ 0 , a quadratic equation in $\varepsilon_{t 0}$ follows which has at most two solutions. Then, $\varepsilon_{t 0}$ can not be a continuous non-degenerate random variable conditioned on $I_{t-1}$ which contradicts with assumption (a).

\section{References}

Baillie R.T., Bollerslev T., Mikkelsen H.O. 1996. Fractionally integrated autoregressive conditional heteroskedasticity. Journal of Econometrics 74: 3-30. 
Bollerslev T. 1986. Generalized Autoregressive Conditional Heteroskedasticity. Journal of Econometrics 31: 307-327.

Bollerslev T. 1990. Modelliing the Coherence in Short-Run Nominal Exchange Rates: A Multivariate Generalized ARCH Approach. Review of Economics and Statistics 72: 498-505.

Bollerslev T., Mikkelsen H.O. 1996. Modelling and Pricing Long Memory in Stock Market Volatility. Journal of Econometrics 73: 151-184.

Chen C-H, Shih H-T. 2002. The evolution of the stock market in China's transitional economy Advances in Chinese economic studies. Edward Elgar Publishing Limited. 37-54.

Engle R. F. 1982. Autoregressive Conditional Heteroscedasticity with Estimates of the Variance of United Kingdom Inflation. Econometrica 50: 987-1008.

Engle R. F., Kroner K. F. 1995. Multivariate Simultaneous Generalized ARCH. Econometric Theory 11: $122-150$

Engel R. F., Ng V. K. 1993. Measuring and Testing the Impact of News on Volatility. The Journal of Finance 48: 1749-1778.

Engel R. F., Ng V. K., Rothschild M. 1990. Asset Pricing with A Factor ARCH Covariance Structure: Empirical Estimates for Treasury Bills, Journal of Econometrics 45: 213-238.

Fornari F., Mele A. 1996. Modeling the changing asymmetry of conditional variances. Economics Letters 50: 197-203.

1997. sign- and volatility-switching ARCH model: theory and applications to international stock markets, Journal of Applied Econometrics 12: 49-65.

Glosten L. R., Jagannathan R, Runkle D. E. 1993. On the relation between the expected value and volatility of nominal excess return on stocks. Journal of Finance 48: 1779-1801.

Kang J., Liu M. H, Ni S. X. 2002. Contrarian and momentum strategies in the China stock market: 1993-2000. Pacific-Basin Finance Journal 10: 243-265.

Kim C-J. 1993. Unobserved-components time series models with Markov-Switching heteroskedasticity: changes in regime and the link between inflation rates and inflation uncertainty. Journal of Business \& Economic Statistics 11. 341-349.

Kim S., Tsurumi H. 2000. Korean Currency Crisis and Regime Change: A Multivariate GARCH Model with Bayesian Approach. Asia Pacific Financial Markets 7. 31-44.

Nelson D. B. 1991. Conditional heteroskedasticity in asset return: a new approach, Econometrica 59: 347-370.

Stout W. F. 1974. Almost Sure Convergence, Academic Press.

Weiss A. A. 1986. Asymptotic theory for ARCH models: Estimation and testing. Econometric Theory 2: $107-131$.

Zakoian J. M. 1994. Threshold heteroskedastic models. Journal of Economic Dynamics and Control 18: $931-955$ 